

\title{
La perception érotique du corps dans l'esthétique épicurienne
}

Julie Giovacchini

\section{To cite this version:}

Julie Giovacchini. La perception érotique du corps dans l'esthétique épicurienne. Le corps humain et les cinq sens: positions du débat dans l'Antiquité, Géraldine Puccini-Delbey, Oct 2011, Pessac, France. pp.91-100. hal-01267482

\section{HAL Id: hal-01267482 \\ https://hal.science/hal-01267482}

Submitted on 4 Feb 2016

HAL is a multi-disciplinary open access archive for the deposit and dissemination of scientific research documents, whether they are published or not. The documents may come from teaching and research institutions in France or abroad, or from public or private research centers.
L'archive ouverte pluridisciplinaire HAL, est destinée au dépôt et à la diffusion de documents scientifiques de niveau recherche, publiés ou non, émanant des établissements d'enseignement et de recherche français ou étrangers, des laboratoires publics ou privés. 


\section{La perception érotique du corps dans l'esthétique épicurienne}

La situation érotique est une situation esthétique paradoxale. Elle prend racine dans une perception - celle du corps de l'être désiré - qui est par définition une perception perverse, c'est-à-dire fallacieuse.

Dans le cadre de la doctrine épicurienne, le problème ainsi posé est particulièrement délicat, puisque pour Épicure la sensation est supposée rester «toujours vraie ». La perception érotique rejoint alors le corpus des illusions qu'il s'agit de discerner et élucider afin de réhabiliter le premier élément de la canonique sur lequel se bâtit l'ensemble de la noétique épicurienne.

En ce sens, la construction du chant IV du De Rerum Natura est tout à fait cohérente : la diatribe de Lucrèce contre l'amour et ses errements prend place après un très long développement consacré, d'une part, à la théorie épicurienne de la sensation et du simulacre, qui fonde gnoséologiquement le premier élément du canon, d'autre part, à la réfutation des positions sceptiques, en particulier celles rattachées aux modes d'Énésidème qui s'appuient sur la mise en évidence de la relativité de toute perception sensible. La stratégie rhétorique de Lucrèce, qui place le cas de l'amour, perception faussée d'un sujet malade, à la toute fin de son argumentaire, vise, comme nous allons tenter de le montrer, à faire de cette question de canonique un véritable problème d'esthétique au sens moderne ; c'est-à-dire à montrer que la beauté n'est pas une qualité sensible permanente des objets, et que par conséquent la question n'est pas tant de sa réalité que de ses conditions de production et d'émergence ; en d'autres termes, que la perception érotique nous oblige, dans un cadre épicurien, à repenser profondément les rapports qui unissent la poétique et l'esthétique.

\section{Le piège de Vénus}

La question de la nature de la beauté n'est pas absolument absente des textes d'Épicure. Elle apparaît par exemple dans la Sentence Vaticane 61 :

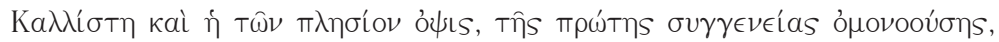

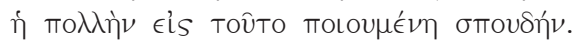

«Très belle, vraiment, est la vue des proches, ceux qui nous sont unis par la première des parentés, elle qui nous fait mettre en œuvre tout notre zèle en vue de cette union. » 
On peut comprendre cette sentence en la rapprochant de ce qui est dit par les épicuriens de la vision des dieux et de leur apparence ${ }^{1}$. La forme des dieux est belle parce qu'elle est proche de la nôtre. Le sentiment du beau naît avant tout d'un sentiment de familiarité. Il s'agit là d'une beauté positive, car elle nous porte à la philia ou bien au sentiment religieux de piété, qui est une autre forme du lien social pour l'épicurisme. Le beau est donc beau en tant qu'occasion de rapprochement, ou plus exactement, c'est le lien qu'il crée entre les êtres qui conditionne l'existence d'un beau identifiable.

Si la proximité naturelle dont il est ici question favorise donc l'élan vers la beauté, celle-ci n'a rien à voir avec celle qui suscite le désir de rapprochement et de communauté beaucoup plus artificiel qui anime l'amant. L'intimité de l'amant et de l'aimé n'est pas sociale ou amicale, c'est-à-dire productrice de liens politiques solides et harmonieux ; mais elle est factice, ne repose sur rien et emprisonne l'amant dans un délire schizoïde.

C'est donc que Vénus façonne un piège, en produisant une pâle imitation de la forme véritablement belle qui rapproche d'autrui. Ce piège est mis en évidence par Lucrèce au chant IV, v. 1153-1170 du De Rerum Natura, dans un passage qui fait état de la distorsion du regard amoureux par l'énumération d'une série d'inversions esthétiques suscitées par le désir :

Nam faciunt homines plerumque cupidine caeci et tribuunt ea quae non sunt his commoda vere. Multimodis igitur pravas turpisque videmus esse in deliciis summoque in honore vigere. Atque alios alii inrident Veneremque suadent ut placent, quoniam foedo adflictentur amore, nec sua respiciunt miseri mala maxima saepe. Nigra melichrus est, inmunda et fetida acosmos, caesia Palladium, nervosa et lignea dorcas, parvula, pumilio, chariton mia, tota merum sal, magna atque inmanis cataplexis plenaque honoris.

Balba loqui non quit, traulizi, muta pudens est; at flagrans, odiosa, loquacula Lampadium fit. Ischnon eromenion tum fit, cum vivere non quit prae macie; rhadine verost iam mortua tussi. At tumida et mammosa Ceres est ipsa ab Iaccho, simula Silena ac Saturast, labeosa philema. Cetera de genere hoc longum est si dicere coner.

Lucrèce reprend ici un thème classique de diatribe : l'inversion des valeurs produite par l'esprit enfiévré de l'amoureux. Ce passage montre avec éloquence comment l'amour fausse la perception des qualités réelles de l'objet désiré par projection d'un voile qui s'ajoute en l'obscurcissant au contenu de la sensation. L'illusion érotique se construit ainsi contre le véritable témoignage des sens, en s'attaquant à son véhicule le plus imprécis et le plus susceptible d'être faussé : la

1 Cicéron, De nat. deor., I, p. 47. 
vue. Par opposition avec le toucher, qui s'appuie sur un contact direct et donc peu modifiable, la vue est le plus indirect des sens. L'image, en tant qu'elle se nourrit de contenus visuels qu'elle interprète, a un pouvoir d'illusion considérable et peut ainsi rendre présent un objet absent - ou, en l'occurrence, recouvrir l'objet présent, en réalité odieux, d'un masque désirable qui aura les apparences d'un être réel. Comme dans le cas de certaines illusions d'optique, l'aveuglement de l'amant vient de sa situation de perception et des canaux par lesquels transite cette perception : l'amant voit trop, de trop près, trop souvent ; il n'est plus en état de ramener son excitation à de plus justes proportions (il refuse par exemple de confronter l'image de l'aimée avec son odeur fétide, alors même que son odorat lui offrirait une appréhension plus directe et par là plus certaine).

L'état amoureux procède finalement pour Lucrèce d'un mécanisme physiologiquement semblable à celui du rêve ${ }^{2}$; l'attention du dormeur comme celle de l'amant se concentrent sur un simulacre qui n'émane pas d'un objet réellement présent, mais de l'âme elle-même, qui construit son propre objet à l'image de son attente et de son désir, et qui choisit ce simulacre comme focale privilégiée. Mais alors qu'il ne nous viendrait pas à l'idée de confondre consciemment les ombres du rêve avec la réalité, l'état amoureux fait perdre toute lucidité, et nous rend semblables à Pygmalion, artisan de sa folie. Le délire érotique, comme le montre le dispositif des qualités inversées, est donc délire de nature métaphorique : Éros modifie, au-delà de la perception, le langage même de l'amant, puisque des termes flatteurs sont substitués à ceux, plus triviaux, qui décriraient avec réalisme l'amante faussement parée ; la construction de l'objet s'accompagne nécessairement d'une construction rhétorique par laquelle l'amant séduit et subverti se trouve à son tour en situation active de séduction et subversion du discours.

Cette position lucrétienne n'est intelligible que dans le cadre bien particulier de la canonique épicurienne, selon laquelle les notions qui ne naissent pas directement de la rencontre directe avec les sensations (periptosis) sont forgées par analogie, ressemblance ou composition (analogia, omoioteta, sunthesis) ${ }^{3}$. Ces trois derniers modes obéissent au modèle de la metabasis, déplacement ou transposition à partir d'un matériel mental déjà présent. Pour les épicuriens, le saut conceptuel que représente la metabasis n'est valable que dans la mesure où il y a bien toujours, au moins en droit, une possibilité d'associer le produit de ce transfert à un support empirique réel. Or, ce n'est pas le cas de la métaphore amoureuse : la beauté n'est pas le produit d'une rencontre empirique effective, mais résulte d'une reconstruction abusive opérée par une dianoia délirante. La beauté perçue par l'amant est un pur artifice.

2 DRN, IV, 757-767 ; IV, p. 1032-1033. Sur la question particulière du rêve érotique, on pourra consulter J. Pigeaud, « Le rêve érotique dans l'Antiquité gréco-romaine : l'oneirogmos ", dans Littérature, Médecine, Société - Rêves et insomnies, 3, 1981 et K. Descoings, "Fantasma d'amore : quand la bien-aimée vient hanter son poète (Antiquité et Renaissance) ", Camenae, 8, 2010. 
Ce qui revient à dire, toujours dans le cadre de la canonique épicurienne, que le beau n'est pas une qualité sensible permanente. En tant que critère du vrai, la sensation signale des caractères réels des objets, qui ne sont pas totalement réductibles aux mouvements imperceptibles des atomes dans le vide. Les atomes eux-mêmes ne possèdent pas de qualités sensibles ; mais les corps, qui ne se réduisent pas aux atomes qui les composent, en possèdent.

Parmi ces caractères « réels », les épicuriens distinguent les sumbebekota (chez Lucrèce conjuncta) qui correspondent à des propriétés permanentes des corps (forme, taille, poids, couleur dans certains cas, odeur, consistance), qui sont inséparables du corps et dont la suppression modifie ce dernier de façon essentielle, et les sumptomata (chez Lucrèce euenta), événements ou accidents contingents de ce corps, qui peuvent être séparés du corps sans dommage pour ce dernier (par exemple l'inscription d'un être dans le temps) ${ }^{4}$. Si la question peut se poser concernant la beauté des dieux, il est évident que la beauté du corps aimé envisagée par Lucrèce dans le texte précédent, en tant qu'elle est totalement dépendante du point de vue de celui qui l'attribue, ne peut faire partie des conjuncta. En tant qu'euentum, au même titre que le temps, elle ne peut donc être rattachée à une quelconque prénotion du beau, qui garantirait la vérité des jugements esthétiques. De même qu'en Her., 72, le sentiment du temps provient de la subjectivité de celui qui l'éprouve (c'est la succession répétée du jour et de la nuit qui imprime dans l'esprit la conscience d'un rythme temporel), de même le sentiment du beau se renforce-t-il par la proximité et l'habitude ; mais il convient encore d'opposer la consistance de ce sentiment lorsqu'il émerge d'une relation de philia telle que celle évoquée dans la Sentence Vaticane 61, et son incohérence absolue lorsqu'il est question d'une relation érotique dans laquelle le lien ne se construit que sur la base d'illusions et de fantasmes. C'est cette dernière distinction qui permet sans doute de comprendre la conclusion relativement modérée du même chant IV de Lucrèce qui clôt les violentes attaques du poète contre l'amour par une sorte d'éloge paradoxal de la conjugalité sereine (v. 1278-1287) :

Nec divinitus interdum Venerisque sagittis
deteriore fit ut forma muliercula ametur.
Nam facit ipsa suis interdum femina factis,
morigerisque modis, et munde corpore culto,
ut facile insuescat secum te degere vitam.
Quod superest, consuetudo concinnat amorem;
nam leviter quamvis quod crebro tunditur ictu,
vincitur in longo spatio tamen atque labascit.
Nonne vides etiam guttas in saxa cadentis
umoris longo in spatio pertundere saxa?

Aux flèches de Vénus, perfides, sont opposés les filets loyaux de l'habitude appuyée sur de véritables qualités, seules capables de tisser un lien solide et non décevant. Un point suggestif demeure : le verbe concinnare, employé

4 Épicure, Her., 72. 
ci-dessus, désigne souvent en latin le piège tressé par l'oiseleur avec des joncs. L'amour demeurerait-elle une relation périlleuse, quand bien même il serait difficile de le distinguer d'une philia toujours désirable?

\section{Une parodie de l'érotique platonicienne}

Lucrèce décrivant la beauté comme un artifice forgé par le désir de l'amant et entravant son jugement semble inverser les thèses développées par Platon sur le rôle protreptique de l'eros. Tandis que chez Platon le désir, aiguillonné par le spectacle de la beauté, peut grâce à cette dernière s'élever progressivement du sensible à l'intelligible et accéder à une beauté intellectuelle qui le libèrera de la tyrannie du corps, chez Lucrèce au contraire le désir perturbe profondément le fonctionnement psychosomatique. L'attention, focalisée sur des simulacres mensongers, se perd dans une hallucination pathologique qui la détourne des véritables fonctions érotiques - à savoir les fonctions sexuelles. Elle transforme alors un désir naturel en appétit désordonné. Le désir n’est donc plus élevé vers le Beau par un objet supérieur ; il crée de toute pièce un objet décevant dont l'obtention sera toujours l'occasion d'un plaisir médiocre en regard des aspirations illimitées de l'amant ${ }^{5}$.

On en revient en réalité avec Lucrèce à une injonction semblable au « connais-toi toi même socratique »; la différence demeure que cette injonction s'adresse chez Lucrèce aux sens, non à l'esprit. Il ne s'agit pas de rentrer en soi-même, mais bien d'en sortir et de retrouver un contact pour ainsi dire épidermique avec l'extérieur de soi, supposé arriver jusqu'à nous par le simulacre. Le corps sentant est interface avec le monde ; mais le désir amoureux brouille cette interface, en transformant le corps en point aveugle capable même de projeter des anti-simulacres ${ }^{6}$.

Mais Lucrèce ne se contente pas de critiquer le modèle platonicien de l'érotique. Il le parodie véritablement, au sens où ce thème de l'aveuglement de l'amant est en réalité déjà présent chez Platon ; ainsi en Rep., 474 d, Socrate décrit explicitement à Glaucon l'aveuglement de l'amoureux. Il s'agit donc véritablement pour Lucrèce de prendre le platonisme au piège de ses propres contradictions internes. Le rôle protreptique de l'eros est une hypothèse soutenue dans le platonisme par une réflexion développée concernant le statut et la forme du discours philosophique, susceptible de favoriser la sublimation du désir. C'est peu ou prou l'objet du Phèdre. Le philosophe doit savoir proposer un objet séduisant susceptible d'éveiller l'appétit ; la recherche poétique du beau est ainsi pour Platon une voie naturelle et justifiée.

5 A. Gigandet parle à ce propos d'un " dispositif d'illusion »: " De l'amour : Vénus de Lucrèce et Erôs platonicien ", dans Rémy Poignault (dir.), Présence de Lucrèce : actes du colloque tenu à Tours, 3-5 décembre 1998, Centre de recherches A. Piganiol, Université de Tours, 1999.

6 Cf. L. Landolfi, « "Didascalica antierotica": "insaniae exempla, mellita cognomina": a proposito di Lucrezio De rerum natura 4, p. 1144-1169 », La Parola del Passato, 64, n 364, 2009. 
La parodie lucrétienne reprend à son compte cet intérêt porté à la forme du discours philosophique. Le De Rerum Natura cherche bien, par la voie poétique, à susciter chez Memmius un éveil philosophique. Il faudra donc que la VenusVoluptas sous l'égide de laquelle il se place constitue à la fois une réponse critique à l'esthétique platonicienne, et une solution satisfaisante à cet épineux problème de la sublimation érotique nécessaire à toute entreprise philosophique.

\section{D'Ovide à Lucrèce : les enjeux d'une réconciliation avec Vénus}

Pour résoudre cette difficulté, il est sans doute nécessaire de faire un bref détour par un autre poète latin : Ovide. Le passage du chant IV envisagé plus haut a un équivalent célèbre dans l'Ars amatoria (II, 651-672), et cette proximité a été déjà abondamment étudiée. Mais comme l'a démontré C. Lévy ${ }^{7}$, il y a aussi de très nombreuses différences, en particulier sur cette question des enjeux intellectuels de l'érotique.

Pour Ovide également, la situation amoureuse pose la question du « connaistoi toi-même ", en invitant à sortir de soi en résistant au piège de l'immersion dans son propre affect. Mais Ovide envisage cette sortie de soi dans le cadre d'une démarche de séduction qui laisse en réalité de côté la possibilité d'un accès au vrai. "Se connaître, c'est savoir rester à la superficie de soi-même, pouvoir contrôler et manipuler l'image qu'on donne de soi $^{8}{ }^{\star}$. Ovide semble prendre acte de l'évacuation de la question du vrai dans la situation érotique, question remplacée sine die par celle du beau : voir le beau, saisir le beau, jouir du beau. L'esthétique ovidienne reprend et pousse jusqu'à l'absurde l'injonction épicurienne d'en rester à la sensation, et par là abolit la distinction entre niveau phénoménal et niveau atomique, et donc toute la question canonique. En ce sens, comme le souligne C. Lévy, Lucrèce et Ovide, à l'issue d'une analyse somme toute très proche de ce qui se passe dans la rencontre érotique, en viennent à s'opposer comme le dogmatisme s'oppose au scepticisme.

Lucrèce veut en finir avec le piège de Vénus d'une part pour préserver la canonique épicurienne du piège du relativisme énésidémien, d'autre part pour redonner une légitimité au plaisir sexuel, qui reste, dans l'horizon de l'hédonisme épicurien, l'objet d'un désir naturel dont il faut restaurer la pureté et l'innocuité ; Ovide n'a pas de telles prétentions, dans la mesure où, d'une part, il considère que la question de la vérité n'a rien à faire avec l'érotique en particulier et l'esthétique en général, et, d'autre part, il envisage le désir sexuel comme un désir resté en "état de nature ", et comme tel profitable, sans qu'il soit besoin de l'encadrer ou de le diriger. Pour Ovide, Vénus est bien un piège

7 C. Lévy, "Aimer et souffrir : quelques réflexions sur la "Philosophie dans le boudoir" de l'Ars amatoria ", dans Laurence Boulègue, Carlos Lévy (dir.), Hédonismes : penser et dire le plaisir dans l'Antiquité et à la Renaissance, Villeneuve-d'Ascq, Pr. Universitaires du Septentrion, 2007.

8 Commentaire d'Ovide, Ars amat., II, p. 501-502. 
que l'amant utilise à ses propres fins pour y faire tomber ses victimes et en jouir. Pour Lucrèce, l'amant lui-même est d'une certaine façon toujours victime de ce piège, mais il est nécessaire, pour que cette chute devienne l'occasion d'un plaisir et non d'une souffrance, de le désamorcer au préalable en le délestant de ses éléments pervers. D'où l'idée, présente dès l'invocation qui inaugure le De Rerum Natura, de présenter Vénus comme un piège en soi innocent, dans lequel il peut éventuellement être plaisant de se laisser prendre. Vénus captive au sens propre les espèces animales, ut cupide generatim saecla propagent : préservées des illusions amoureuses, les bêtes se laissent aller à un instinct de reproduction bénéfique sans souffrir davantage de dommages. La diatribe contre l'amour est elle-même étoffée de considérations techniques concernant la physiologie de la reproduction, qui identifient le plaisir féminin à un autre de ces pièges nécessaires pour assurer la survie des espèces.

L'erreur de l'amant consiste donc à fonder sa recherche érotique sur des critères inadéquats puisqu'il préfère, à la réalité substantielle du plaisir sexuel, les fantasmes ténus forgés par sa propre rhétorique complaisante. La beauté ne peut donc être utilisée comme critère ; mais elle peut être soumise à un critère véritable, à savoir le pathos qu'elle engage : quel plaisir et quelles souffrances retire-t-on de l'objet désiré ? Critère qui peut également se révéler pertinent pour penser la beauté du poème lui-même. Le piège de la beauté est en ce sens le piège dans lequel la beauté elle-même se laisse prendre, soumise à la juridiction de la recherche de l'utile, utile « naturel » (la propagation des espèces) et «social» (propagation de la doctrine épicurienne).

L'individu a toujours besoin de croire à la beauté de l'objet de son désir, afin de justifier la focalisation de son attention (sur une personne ou une doctrine). L'habitude renforce alors l'illusion par l'incrustation mentale des simulacres érotiques, qu'elle soit construite par la vie commune avec l'être aimé ou par l'assimilation quotidienne des résumés de la doctrine. Le pathos qui en résulte est bon (agréable), et il vient à rebours justifier le recours à la « saine » illusion, illusion qui guérit au lieu de rendre malade.

\section{La poétique au risque de l'esthétique}

Quelles seront donc, dans ce cadre et avec ces enjeux, les règles d'une poétique proprement épicurienne ? On relèvera, pour commencer, le privilège évident qu'ont acquis au sein de l'école épicurienne certains supports esthétiques par rapport à d'autres. Il n'est pas anodin que Philodème de Gadara par exemple ait proposé une réflexion sur la poésie et la musique, et qu'à côté de cela les références aux arts picturaux soient remarquablement absentes, en tant que sujets de réflexion, du corpus épicurien ${ }^{9}$.

9 Cf. en particulier E. Asmis, « Epicurean poetics », dans John J. Cleary (dir.), Proceedings of the Boston Area Colloquium in ancient philosophy, VII, 1991, Lanham, Md. \& London, University Pr. of America, 1993. 
Musique et poésie sont en effet des arts qui sollicitent l'ouie et non la vue. Puisque le son est un corps, les sensations auditives sont des sensations directes.

Le poète philosophe doit ainsi se garder de prendre son modèle chez le peintre. Il se méfie de l'image et en évite les travers. Il imite en cela la beauté de Vénus décrite au début du poème :

- beauté de la clarté, de l'évidence, de la lumière, qui apparaît dans sa nudité et ne déçoit pas ;

- non pas beauté spectaculaire qui se contemplerait de loin, mais beauté qui se goûte, se respire, se caresse, comme le miel, le parfum, la peau.

Vénus n'est pas une image : imiter Vénus, ce n'est pas créer un simulacre de plus, mais imiter son pouvoir érotique et présenter au lecteur, identifié à Mars, un corps adorable que l'on ne peut que vouloir toucher, dévorer, s'approprier ; ce même lecteur, enivré par ce contact avec la chair de Vénus, se laissera alors volontiers prendre aux filets de la doctrine.

C'est bien là tout le sens d'une distinction rapportée par Plutarque entre épicuriens et cyrénaïques :

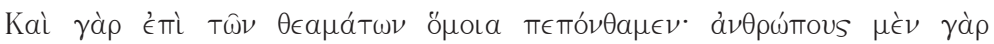

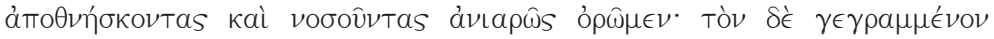

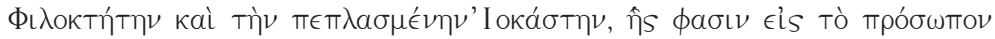

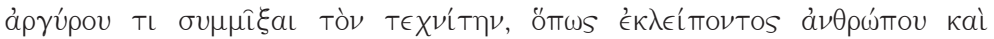

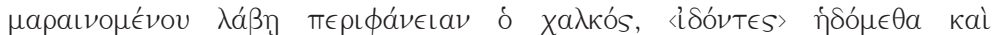

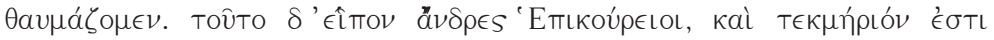

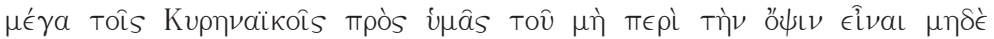

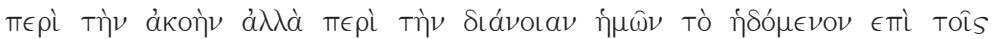

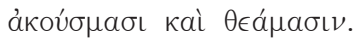

«Et, de fait, nous ressentons les mêmes choses devant des représentations théâtrales. Car si habituellement nous regardons avec horreur les mourants et les malades, en revanche devant un tableau qui représente Philoctète, ou cette statue de Jocaste que, d'après ce que l'on dit, le sculpteur avait recouvert d'une couche d'argent afin que la teinte du bronze évoquât la défaillance et l'épuisement, nous éprouvons du plaisir et de l'admiration. Et c'est bien, messieurs les Épicuriens, dis-je, un grand argument que les Cyrénaïques vous opposent, pour établir que notre sensation de plaisir auditif ou visuel ne vient pas de la vue ni de l'ouie, mais du raisonnement ${ }^{10}$. »
\end{abstract}

Dans ce passage, le plaisir est décrit en des termes qui rappellent évidemment la définition aristotélicienne du plaisir mimétique ${ }^{11}$. C'est la conscience de la représentation qui provoque pour Aristote le plaisir du spectateur, la jouissance provenant du décalage entre l'objet réel et l'objet mimé. Ce décalage est perçu par un raisonnement ; le plaisir n'est donc pas suscité directement par l'objet mais par la dianoia dont cet objet est l'occasion.

10 Plutarque, Quaest. Conu., 674a-b.

11 Poet., 4, 1448b, p. 15-17. 
Si Plutarque dit vrai et si cette conception du plaisir mimétique a bien été reprise par les cyrénaïques et critiquée par les épicuriens, il s'agit là d'un fait surprenant : pourquoi un tel refus chez les épicuriens ? Pensons au célèbre prélude du chant II, le " suave mari magno ${ }^{12}$ " : ne s'agit-il pas de la description d'un plaisir mimétique produit par le décalage entre ce qui est perçu (la condition pénible des hommes soumis aux aléas de l'existence) et la situation paisible du sage qui contemple ce spectacle du haut de son promontoire?

En réalité, cette opposition des cyrénaïques et des épicuriens se comprend si on la nourrit d'une source plus consistante : les textes de Philodème de Gadara sur la musique et sur la poésie. Philodème y rapporte les différentes critiques formulées par les épicuriens contre d'une part les kritikoi, théoriciens de la musique qui détournent l'art de sa fonction véritable de divertissement pour en faire un élément de paideia, ce que refusent les épicuriens pour qui la seule paideia valable est la philosophie ; d'autre part les formalistes qui évaluent l'œuvre d'art en fonction de critères d'euphonie et de style, selon un présupposé qui fait résider la qualité du poème ou de la musique essentiellement dans son apparence phénoménale. Or les sons en eux-mêmes pour Philodème ne peuvent donner de plaisir, seul le sens en est capable ${ }^{13}$.

Les épicuriens se battent sur cette question sur deux fronts simultanés. D'une part, ils récusent toute esthétique visant à laisser dans l'ombre le rôle de la dianoia: la beauté sensible n'est pas une réalité suffisamment solide pour être ainsi utilisée comme critère de jugement, et elle n'est pas en elle-même susceptible de quoi que ce soit de valable si elle n'est pas mise en relation par un jugement, même primitif, avec un pathos agréable consistant. D'autre part, ils refusent également que ce nécessaire jugement esthétique fonctionne par comparaison avec une rationalité extérieure à l'œuvre elle-même. Pour Philodème, le beau poème, la bonne musique contiennent déjà du rationnel, et c'est de l'identification et de l'appropriation de ce rationnel que provient le plaisir. Si l'on revient au "suave mari magno ", Lucrèce lui-même établit une hiérarchie entre le plaisir mimétique qui procède d'une telle perception de décalage, et un plaisir plus doux et sans doute plus authentique qui consiste à vivre directement la vie du sage - vie par laquelle le contenu rationnel de la nature et de la condition humaine deviennent explicites et donc évidents.

Dans les textes esthétiques de Philodème, la position épicurienne a des conséquences tout à fait remarquables, et en particulier celle-ci : l'absence de la notion de beauté. Celle-ci n'est citée que pour être récusée, rabattue sur l'utile, le rationnel ou le plaisant qui, comme dans le cas du chant IV du De Rerum Natura, la détrônent ou en tout cas modifient considérablement sa compréhen-

12 DRN, II, p. 1-61

13 Cf. De Poematis, V, XXIX, p. 24-35 et XXVI, p. 1-10. Ces textes sont commentés par D. Armstrong, " Philodème et l'appréciation de l'effet poétique par l'intellect: (Poèmes V et Herc. 1676) », dans Clara Auvray-Assayas et Daniel Delattre (dir.), Cicéron et Philodème : la polémique en philosophie, Paris, Éd. Rue d'Ulm, 2001. 
sion. Philodème construit sous nos yeux une poétique qui fait magistralement l'économie de la question du beau en tant que question de forme ou d'objectif. Lucrèce quant à lui nous offre un exemple de cette poétique " en acte ». La poésie devient un certain usage du discours, susceptible de devenir usage thérapeutique une fois qu'on est sorti du piège de l'image et de la séduction qui s'y rattache. L'extrême sensualité que permet l'écritude poétique est une illustration rêvée de la volonté de la part du poète épicurien de proposer un texte susceptible de mener le lecteur à une conversion effective vers la philosophie. La poésie érotique agit comme un simulacre discursif, et son efficace doit être pensée sur le modèle de celle du rêve sensuel ; si rêver un acte sexuel est déjà un acte sexuel, désirer la Vénus-Voluptas de papier du De Rerum Natura, c’est déjà rejoindre le cercle d'Épicure.

Julie Giovacchini CNRS-UPR76 